\title{
Zur Geschichte der iatrogenen Krankheiten
}

\author{
Von Erwin H.Ackerknecht (Zürich)
}

Im Laufe des letzten Jahrzehnts sind mindestens ein halbes Dutzend Bücher und unzählige Artikel über die «iatrogenen Krankheiten» erschienen. Die Thalidomidkatastrophe hat nur einem breiteren Publikum zum Bewußtsein gebracht, was Ärzte schon lange wußten, nämlich, daß gerade wirksame Heilmittel außerordentlich gefährliche Nebenerscheinungen haben können. Als iatrogene Krankheiten sind z.B. solche der Haut, des Bluts (besonders seit W.Schultz 1922 die Agranulocytose beschrieben hatte), der Leber, der Niere, des Magen-Darm-Kanals, von Auge und Ohr sowie endokrine Störungen, solche des Nervensystems inklusive Seele, und des Elektrogleichgewichts beschrieben worden. Auch iatrogene CollagenKrankheiten, Infektionen und Malignome sind bekannt. Ursächlich sind auch immer noch so alte Mittel wie Quecksilberverbindungen, Digitalis, organische Arsen- und Goldverbindungen sowie sogar der Sauerstoff am Werk. Schon seit Jahrzehnten haben sich Bestrahlungsschäden, solche durch Salicylate und Transfusion bemerkbar gemacht. Der größte Teil neuerer iatrogener Erkrankungen ist wohl im Zusammenhang mit der sonst so nützlichen und außerordentlich weitverbreiteten Verwendung der Sulfanilamide und der.Antibiotika berichtet worden. Zahlreiche pathologische Nebenwirkungen werden auch auf die Corticosteroide zurückgeführt. Die Tranquilizers sind keineswegs so harmlos, wie ursprünglich angenommen wurde. Das Chlorpromazine ist z.B. in einzelnen Fällen verantwortlich gewesen für so verschiedenartige Erkrankungen wie Parkinson, aplastische Anämie, Icterus und Arythmien. Auch die Antihypertonika, besonders die Ganglion blockierenden, können pathogen sein. Dasselbe gilt für die Anticoagulantien, die anticonvulsierenden, die antirheumatischen und die Kontrastmittel. Schließlich geht man natürlich bei den Cytostatica ebenfalls nicht geringe Risiken ein. Das ist in ganz flüchtigen Umrissen das Bild, das sich aus einem Studium der Literatur über iatrogene Krankheiten, wie etwa D.H.SpaIN's, The complications of modern medical practice (New York 1963), aus den letzten Jahren ergibt.

Die iatrogenen Krankheiten sind aber an sich alt, wahrscheinlich genauso alt wie die Therapie selbst. Sie sind auch längst bekannt. Was neu ist, ist 
ihr wahrscheinlich häufigeres Vorkommen im Zusammenhang mit dem Aufkommen wirksamerer Heilmittel. Neu ist auch das große Interesse an dem Gegenstand; neu ist schließlich der Terminus, der die schon genannten Faktoren und die größere Wissenschaftlichkeit unserer Medizin widerspiegelt.

Besonders berühmte Fälle von iatrogener Krankheit und Tod werden von Louss Lewin in seiner Geschichte der Gifte genannt, wie der Tod von Neros Ritter an Kanthariden, der Ottos II. an Aloe, der des Gregorius an Kolchizin, des Avicenna an einer Kombination von Pfeffer-Klistier und Opium. Auch Heinrich I. und Amalrich wurden durch Abführmittel dahingerafft, während Voltaires Tod dem Opium zugeschrieben wurde. Es ist ja auch ganz offenkundig, daß der jahrhundertelange Gebrauch und Mißbrauch nicht nur von Medikamenten besonders zum Abführen und Erbrechen, sondern gerade des Aderlasses immer wieder fatale Folgen haben mußte. Als iatrogene Krankheiten muß man wohl auch die zwei Seuchen bezeichnen, welche bis zur Mitte des vorigen Jahrhunderts in den Spitälern wüteten: der Flecktyphus, vor allem auf den inneren Abteilungen, die Sepsis in Chirurgie und Geburtshilfe. LeIBNIz nannte darum ja auch die Spitäler seiner Zeit Seminaria mortis.

Die Ärzte haben sich angesichts dieser Tatsachen auch seit Hippokrates über die Alexandriner und Asklepiades bis zu GaLen kritisch über einzelne Behandlungsmethoden ausgesprochen. Auch die alten Inder kannten bereits die schädlichen Folgen von übermäßigem Aderlaß, Abführmitteln und Emetika. Auch im Mittelalter, dem arabischen sowohl wie dem westlichen, kennen wir solche kritischen Äußerungen. Pietro d'Argellato wußte z.B. bereits über die schädlichen Folgen der jodhaltigen Kropfmittel zu berichten. Natürlich handelt es sich infolge des unentwickelten Zustandes der Medizin dieser Epochen und auch später bei diesen Erkrankungen nur teilweise tatsächlich um iatrogene Schäden.

Ausführungen über die schädlichen Nebenwirkungen von Heilmitteln werden in der Neuzeit immer zahlreicher. Im 16. Jahrhundert kennen wir die heftige Polemik des Paracelsus gegen die zeitgenössische Therapie, nicht zuletzt mit Quecksilber. Gleichzeitig beginnen mit FerNelius im 16. Jahrhundert sich die sogenannten Antimercurialisten gegen den Mißbrauch der Quecksilberbehandlung bei der Syphilis zu wenden. Dieselbe Tendenz kann auch im folgenden Jahrhundert beobachtet werden. Der vielseitigste Kritiker iatrogener Erkrankungen in jenem Jahrhundert ist wohl van Helmont. Aber z.B. auch Sydenham, der doch bei der Verwen- 
dung des Quecksilbers bestimmt nicht kleinlich war, weiß andererseits, daß Hysterie und Wassersucht im Gefolge zu starker Aderlässe und Purgationen auftreten können. Besonders agressiv wird (zum Teil grundlos) im 17.Jahrhundert auf die schädlichen Wirkungen der peruanischen Rinde hingewiesen (Gideon Harvey und andere). Es ist bezeichnend für den Geist des Jahrhunderts, daß EтtмüLler 1661 seine Schrift über die sogenannten Präzipitanzien, unter denen sich auch die Chinarinde befand, nicht eine Schrift über den Gebrauch der Präzipitanzien, sondern über den Gebrauch und Mißbrauch der Präzipitanzien nannte.

Am Beginn des 18. Jahrhunderts glaubt BoerhaAve Tobsucht auf übertriebenen Aderlaß und Purgationen zurückführen zu müssen, während er einzelne Skorbutfälle durch den übermäßigen Gebrauch der Chinarinde erklärt. Auch sind die Klagen über schädliche Folgen des therapeutisch verwendeten Phosphors und Arseniks im früheren 18. Jahrhundert so zahlreich, daß diese Medikamente zeitweise nur noch wenig gegeben wurden. GAUB, der Nachfolger Boerhaaves, hat in seiner Krankheitslehre ein ganzes Kapitel über den «unzeitigen Gebrauch der Arzneien».

Anfangs des 18. Jahrhunderts kommt es sogar zur Entstehung von Monographien über iatrogene Krankheiten. Da ist erst einmal der Hallenser Kliniker G.E.STAHL zu nennen mit seiner Untersuchung der übel kurierten und verderbten Krankheiten aus dem Jahre 1726. Stahl verurteilt hier Abführmittel, Brechmittel und Sudorifika im allgemeinen sowie besonders das Opium, auf dessen Mißbrauch er hektische Fieber, Wassersucht, Kachexie, Kolik und Gicht zurückführt. Auch Quecksilber und diverse Epilepsiemittel hält er für recht schädlich. Die Goldmedikation hält er für Schwindel und die Panazee Chinarinde für äußerst gefährlich. Auch die Gesundbrunnen werden zu seiner Zeit seiner Meinung nach sehr mißbraucht, sowie Adstringentien und Eisenmedikation.

Sein sonst ihm so unähnlicher Exfreund, Kollege und Konkurrent in Halle, Friedrich Hoffmans, vertritt dieselben Auffassungen. In seinen Opuscula von 1736 stehen drei diesbezügliche Abhandlungen; die erste behandelt «die unkluge Medikation als Ursache vieler Krankheiten und des Todes». Hoffmann diskutiert hier die falsche Verwendung des Aderlasses, der Vomitorien, der starken Abführmittel, der Schwitzmittel, das Opium und der Quecksilberpräparate. Genau wie Stahl verurteilt er die Adstringentien, weil sie die Blutflüsse, z.B. den ihnen so besonders teuren Hämorrhoidenfluß stoppen und das Chinin. Er glaubt, daß Ärzte wegen ihres zu starken Temperamentes falsche Medikamente verwenden. In einer zweiten 
Abhandlung «Über den Mißbrauch und die Schäden bei der Verwendung gutartiger Heilmittel» geht er noch weiter, oft zu weit. Wasser, Wein, Süßigkeiten, Milch, heiße Infusionen und kalter Trunk können schaden. Dasselbe gilt für die Unterdrückung von Nasenbluten und die Schmerzunterdrückung beim Rheuma. Tee, Kaffee und Schokolade können ebensosehr schädigen wie schweres Essen bei Fieber, Fleisch bei Kopfverletzungen, Süßes bei Magenleiden (dies war übrigens schon vor ihm beobachtet worden). Weitere Schäden können entstehen durch die Verwendung von Milch, Ölen, Fetten, Fußbädern, Bädern, Neutralsalzen, Säuren, Spirituosen und Moschus. In einer dritten Abhandlung beschäftigt er sich mit den «gewöhnlichen Irrtümern in der Anwendung von Lokalmitteln in der Praxis».

Halle scheint überhaupt im 18. Jahrhundert ein Zentrum des Studiums der iatrogenen Krankheiten gewesen zu sein. Es sind zu diesem Thema dort auch eine ganze Reihe von Dissertationen erschienen wie die von G. E. WeIsS aus dem Jahre 1728 über Die Ärzte als Krankheitsursache, in der vor allen Dingen an Hand von Krankengeschichten Aderlaß- und Antimonschäden analysiert werden; fernere derartige Dissertationen wurden verfaßt von Zweifel 1701, Curtius 1714, Zeys 1722, Langguth 1739, Kühne 1763 und Schlenther 1777. Auch J.Lenhard, der in Leipzig 1788 seine Arzneymittel ohne Maske veröffentlichte, hatte in Halle studiert. Größen der Halleschen Fakultät waren bekanntlich J.CH. W.Juncker und J.CH. REIL, welche beide in ihren allgemeinen Werken den iatrogenen Schädigungen oder, wie man früher sagte, Nebenwirkungen der Therapie (1791 und 1799) nicht wenig Aufmerksamkeit schenkten. August Friedrich Hecker, der dasselbe in seiner allgemeinen Pathologie von 1791 tat, war in Halle ausgebildet.

Diese Hallische Tradition setzt sich auch noch ins nächste Jahrhundert fort mit Curt Sprengel, der in seinem Handbuch der Pathologie (Leipzig 1802) sich ausführlich mit dem «Mißbrauch der Arzneien als Gelegenheitsursache der Krankheiten» beschäftigt, wobei Anklänge an Stahl unverkennbar sind, aber noch die inzwischen aufgekommenen Arzneimittel wie das Kirschlorbeerwasser, Nachtschatten, Bilsenkraut, Schierling und Digitalis dazukommen. Natürlich hat sich das Studium der iatrogenen Krankheiten nicht allein auf Halle beschränkt, z.B. erschien im Jahre 1775 in London ein Buch von Thомаs Withers aus Edinburgh, Beobachtungen über den Mißbrauch von Medikamenten, in dem Withers die schädlichen Folgen des Mißbrauchs von Aderlaß, Brechmitteln, Purgieren, Schwitzmitteln, sogenannten Stimulantien, Quecksilber, Opium, Tee, Kaffee, Chinarinde kri- 
tisiert. Im Gebrauch der Kanthariden hingegen ist er eher unkritisch eingestellt.

Auch im 19. Jahrhundert hat man sich natürlich mit iatrogenen Krankheiten beschäftigt. So werden bereits in den zwanziger Jahren sowohl therapeutische Brom- wie therapeutische Jodvergiftungen beschrieben. Andere neue Drogen werden ebenfalls in dieser Richtung erforscht oder die toxische Wirkung älterer Mittel untersucht. Z.B. hat sich der deutsche Kliniker Kussmaul wiederholt solchen Problemen zugewandt, wie im Falle des Quecksilbers und des Aderlasses. Andererseits haben wir nun für eine längere Periode keine allgemeinen Werke zu diesem Thema finden können. Auch verschwinden die entsprechenden Kapitel aus den allgemeinen Werken über Pathologie. Erst Ende des Jahrhunderts beginnt mit dem Buche des unvergeßlichen Louis Lewin, Die Nebenwirkungen der Arzneimittel (Berlin 1881), wieder die Literatur über iatrogene Krankheiten als solche, unter dem damals üblichen, darum von Lewin verwandten und noch für lange vorherrschenden Namen der «Nebenwirkungen». Warum die allgemeinen Werke über iatrogene Krankheiten für längere Zeit verschwunden waren, und ob diese Lücke mit dem therapeutischen Nihilismus zusammenhängt, vermag ich nicht zu entscheiden. Gegebenenfalls kann übrigens, wie HaNs Fischer bemerkt, paradoxerweise sogar der therapeutische Nihilismus Ursache einer iatrogenen Krankheit sein. Auf jeden Fall fällt Lewins Auftreten mit einer starken Aktivierung der Medikationen zusammen.

Lewin versucht erst eine allgemeine Theorie der Nebenwirkungen zu geben (bedingt durch Individuum, zeitlich und örtlich, und durch Beschaffenheit des Mittels). Er stellt fest, daß Mittel, die wirken, eben auch Nebenwirkungen haben, daß solche aber durch chemische Veränderungen der Mittel eingeschränkt werden können. Er wendet sich dann der Einzeluntersuchung der Mittel zu, wobei er als Einteilung die altmodische in Tonika, Adstringentia, Alterantia, Narkotika usw. benützt. Seine Liste der Nebenwirkungen von Medikamenten ist natürlich gleichzeitig eine Liste der damals gebräuchlichsten Medikamente und darum auch aus diesem Grunde interessant. Besonders starke Nebenwirkungen werden nach ihm (er hat die ganze Literatur durchgearbeitet) bei Chinin und Salicylaten beobachtet. Bei Strychnin und Terpentin spielen sie eine geringe Rolle, während Copaiva-Balsam und Wismuth wieder mehr unerwünschte Folgen zeigen. Ganz besonders verbreitet ist der Argyrismus in Folge des damals häufigen Gebrauchs von Silberpräparaten bei Epilepsie und Tabes. Blei, 
Zinkoxyd und Chlorbarium (damals ein «Spezifikum» gegen Skrofulose) zeigen nur mäßige Vergiftungserscheinungen. Dasselbe gilt für das Ergotin (damals hauptsächlich gegen Fibromyoma verwendet).

Besonders verbreitet war natürlich damals der Carbolismus. Carbolsäure wurde auch internistisch verwendet: Senator injizierte es ins Gelenk bei Polyarthritis. Ebenso wurden zahlreiche therapeutische Vergiftungen mit Quecksilber, Arsen und Jod und Brom (vor allem der Bromismus der Epileptiker) berichtet. Auch bei Opium und Belladonna wurden zahlreiche Nebenwirkungen beklagt. Weniger hingegen bei Kampfer, Aconit, Veratrin, Colchizin und Digitalis. Eine häufige Quelle unangenehmer Erscheinungen waren das Chloralhydrat, das Chloroform und der Äther. Der Brechweinstein (damals immer noch als Salbe auf das Haupt von Verrückten geschmiert) konnte als Herzgift agieren. Auf unangenehme Nebenwirkungen mußte man sich auch bei Santonin, Pilokarpin, Canthariden (Blasenpflastern) und Chrysarobin gefaßt machen. Es wird dem aufmerksamen Leser nicht entgangen sein, daß ein recht hoher Prozentsatz dieser Substanzen in den letzten neunzig Jahren aus dem praktischen Gebrauch verschwunden ist.

Das nächste Werk über die Nebenwirkungen der modernen Arzneimittel stammt von Отто SeIfERT und ist in Würzburg 1915, ein Nachtrag 1922 erschienen. Die Einteilung ist hier eine wesentlich modernere in Antipyretika, Anästhetika, Herzmittel, Magen-Darm-Mittel usw. usw. Wir können hier nur über einige Angaben aus diesem Werk berichten. Wieder, wie schon bei Lewin, spielen die Schäden durch Antipyretika eine große Rolle. Während 1881 aber hauptsächlich die Salicylate die Schuldigen waren, sind es jetzt Antifebrin, Antipyrin, Phenacetin und Pyramidon. Bei den Anästhetika stehen Bromäthyl (mit Leberschädigungen), Kokain, Chloroform, Sulfonal und Veronal im Vordergrund. Bei den Herzmitteln ist es die Digitalis, bei den Magen-Darm-Erkrankungen das Oleum chenopodii (Emetin macht nur Verstopfung), bei den Harnerkrankungen das Salol. Um eine Diskussion des Salvarsans drückt sich der Verfasser. Bei den Hautkrankheiten warnt er vor Kakodyl (auch gegen Tuberkulose viel verwendet) und Aroxyl, ferner beschäftigt er sich ausgiebig mit den Jodpräparaten, mit Kollargol und Lysol. Im zweiten Band, der nach der Grippe von 1918 erschien, hat er dann auch Gelegenheit über die zahlreichen verheerenden Schädigungen durch Optochin zu berichten.

Der Altmeister der deutschen Klinik, StrüMPELL, hat dann 1922 in der deutschen Medizinischen Wochenschrift ebenfalls zu unserem Thema Stel- 
lung genommen. Nach dem therapeutischen Nihilismus herrsche nun wieder eine therapeutische Hochflut. Strümpell wendet sich gegen die Polypragmasie jener Zeit und bezeichnet als besonders häufig schädliche Nebenwirkungen verursachend das Sublimat, die Karbolsäure, das Jodoform, das Salvarsan, das Optochin und das Sulfonal. Die Hypochondrie könne auch eine Art iatrogener Erkrankung sein, indem sie von gewissen Ärzten geradezu gezüchtet wird.

Es ist unmöglich, sich hier mit all den Detailstudien und Diskussionen zu beschäftigen, die in jenen Jahrzehnten über die Nebenwirkungen des Tuberkulins, des Insulins, des Mesothoriums usw. usw. stattgefunden haben. Eine dritte Welle der Beschäftigung mit den iatrogenen Krankheiten als solchen beginnt dann in den fünfziger Jahren, wie wir schon anfänglich ausgeführt haben. Es ist in letzter Zeit von Autoren auf diesem Gebiet besonders unterstrichen worden, daß Präventivmaßnahmen im allgemeinen wesentlich weniger Gefahren iatrogener Erkrankungen in sich bergen als therapeutische Eingriffe. 